\title{
It's Counting That Counts
}

\author{
Michael N Marsh ${ }^{*}$ \\ Oxford University, UK \\ *Corresponding author: mikemarshmd@uwclub.net
}

Cite This Article: Michael N Marsh, "XIt's Counting That Counts.” International Journal of Celiac Disease, vol. 4, no. 1 (2016): 1-3. doi: 10.12691/ijcd-4-1-5.

In the last issue of the journal, Siriweera and colleagues [1] revisit the controversial area of inter-epithelial space lymphocyte (IEL) "counting" in regard to the diagnosis of celiac disease (or, as I prefer, for good reason, gluten sensitivity). Their histologically-based approach is a further attempt to re-determine the diagnostic validity of this method of enumerating IEL. It was based on another definition of the 'control' upper limit for normal mucosae, but also to 'assess the diagnostic accuracy of existing criteria for IEL in diagnosis of celiac disease'.

Briefly, based on $\mathrm{x} 400$ examinations of $3 \mu \mathrm{m}$ H\&E sections of formalin-fixed tissue, their upper limits for control, and celiac, mucosae were 4/100 and 20/100 enterocytes, respectively. These values are exceptionally low for controls, while the ranges for each series were not given, so the extent of their overlap cannot be inferred. Conversely, the data published from our laboratory [2], based on strict morphometric criteria using oil-immersion optics with $1 \mu \mathrm{m}$ toluidine blue-stained Epon sections yielded $95 \%$ confidence limits of 5-27, and 14-61, respectively. Despite that attention to detail, there was a clear overlap between each nominal population, thus reinforcing the obvious conclusion that there is no magical "cut-off" point, because the lymphocyte response (like weight, blood pressure, height, acid secretion) is a graded characteristic [3]. That indicates that there never was, nor could be, clear-cut divisions, as if the two IEL populations (control and celiac) represented separate, bimodal populations. The results of every study published are also confirmatory of that truth, too, but is contrary to Lerner and Matthias [4].

Apart from a few references, there was no in-depth engagement by Siriweera with the considerable literature on IEL, and one which still remains unresolved, as is clear from the recent papers in this journal by Pena, and by Lerner \& Matthias (and see similar recent explorations in Gastroenterology Hepatology: from Bed to Bench, 8(4), 2015). I was also rather surprised that Siriweera's counts were made on 'uninterrupted sections of epithelium comprising 500 enterocytes' which, in my experience, would be very difficult to achieve for normal mucosae and even more so for damaged specimens. Neither do they grade their celiac mucosae (whether one believes the socalled Marsh Classification [5] - albeit modified by so many others - or not).

Now, that is very important because with the very early lesions, histological diagnosis may be well-nigh impossible, especially if the villi are tall and non- infiltrated (Marsh 0). As I was both a practising gastroenterologist and a laboratory-based investigator, it was always clear to me that the histology is but an aid to diagnosis, the latter being ultimately made, with all other relevant data weighed, at the bedside by the clinician in charge. Moreover, diagnosis is difficult in the early stages of evolving celiac disease irrespective of the diagnostic approach utilised [6]. This problem is diagnostically magnified by the current epidemic of so-called noncoeliac gluten sensitivity. This dilemma illustrates the fragile confirmation of coeliac disease in its earliest stages of development, and irrespective of the sophistication of the laboratory tests employed. My own view is that Siriweera's paper hardly contributes to this important debate, and certainly does not help in clarifying any of the issues arising.

So, what are the issues arising? Many have been nicely revisited by Lerner \& Matthias [4]: (i) effects of age on IEL (ii) presence of infections or parasites (H pylori, Giardiasis) (iii) genetic background (iv) geographic, environmental and cultural influences (v) role of other methodologies, including flow cytometry. These authors ask for a standardised methodological approach for enumerating IEL, but that is seemingly an impossible request, since as noted above, there have been innumerable papers on this subject without the slightest attention given towards a universallyagreed approach. Neither the official guidelines published by the American Gastroenterological Association nor the British Society of Gastroenterology have elucidated a preferred modus operandi. And as far as mucosal classification goes, the BSG paper [7] recommends any of the approaches available. One can conclude that neither the authors invited, nor the Organisations' representatives, acknowledged that this allegedly important histological tool employed widely should have been standardised and accepted by everyone.

One recent advance which does require attention, however, has seen the grouping of all mucosal specimens (Marsh 0, I and II) into the category termed by Rostami as "Microscopic Enteropathy" (The Bucharest Consensus) [8]. Here it is recognised, and quite rightly, that a list of differential diagnoses obtains for each mucosal category, thereby overcoming the somewhat ridiculous view that, for example, Marsh I and II lesions represent "nonspecific" appearances. But there is no such histological entity as "non-specific": every tissue either reflects its physiological or pathological state when sampled. It is often scarcely recognised that the Marsh III lesion is 
equally "non-specific" when based on that same presupposition, but requires evaluation among all the other possibilities, in the context of the patient under investigation.

Likewise, the universal trend of referring to the process of villous effacement as the result of "atrophy" is patently incorrect. We have no good conception as to how the mucosa flattens, but it is certainly not due to atrophic process, and individual villi do not progressively become flat until they reach the level of the crypt openings: indeed, histological appearances render that possibility redundant. Neither should it be forgotten that until recently, a regenerating mucosa was a late diagnostic sign of gluten sensitisation. There could be no possibility of subsequent re-growth of villi if they had previously been subject to atrophic degeneration. That makes no sense whatsoever.

But let me return to the actual method of "counting" IEL, and some of the technical problems underlying that process. First, as mentioned above in regard to Siriweera's paper, enterocytes are not regularly arrayed on the villous surfaces in straight lines, or side-by-side like soldiers on parade (Figure 1). Yet it seems to be universally believed that such an arrangement actually occurs. Conversely transmission and scanning EM have shown that enterocytes are distributed in short-range hexagonal groupings. With the precise measurements of cell and nuclear diameters deriving from those studies, an idealised epithelium can be constructed (Figure 2). If notional sections are drawn through it, it becomes quickly observable that for any section made, 50\% enterocyte nuclei (which are necessarily employed in making IEL counts), never appear in any given section. That underscoring of enterocyte nuclei accounts for the spurious twofold over-estimation of IEL counts per 100 enterocytes obtained by the Ferguson technique [2,9].
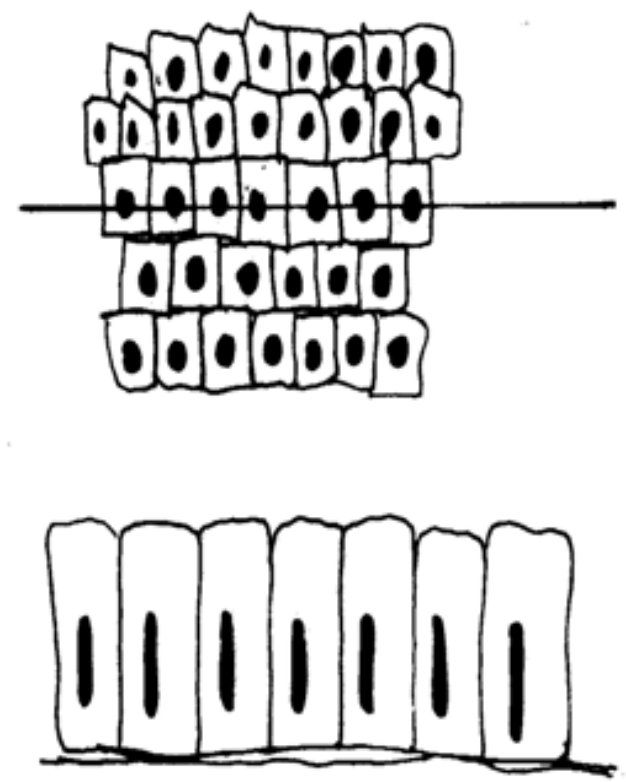

Figure 1. Enterocytes are not positioned along the basement membrane in a linear fashion, as illustrated in the upper display. Therefore, counts of 'enterocytes' (lower diagram) cannot be accomplished as easily as some Methods Sections, as reported in many papers, might suggest. This model obviously predicts that it would be possible to observe large tracts of enterocytes without their nuclei. But that is never encountered in practice. Nevertheless, one could be tempted, while working at the microscope, to think that adjacent nuclei meant that strips of adjacent enterocytes were always being counted. That conceptualisation would likewise be wrong

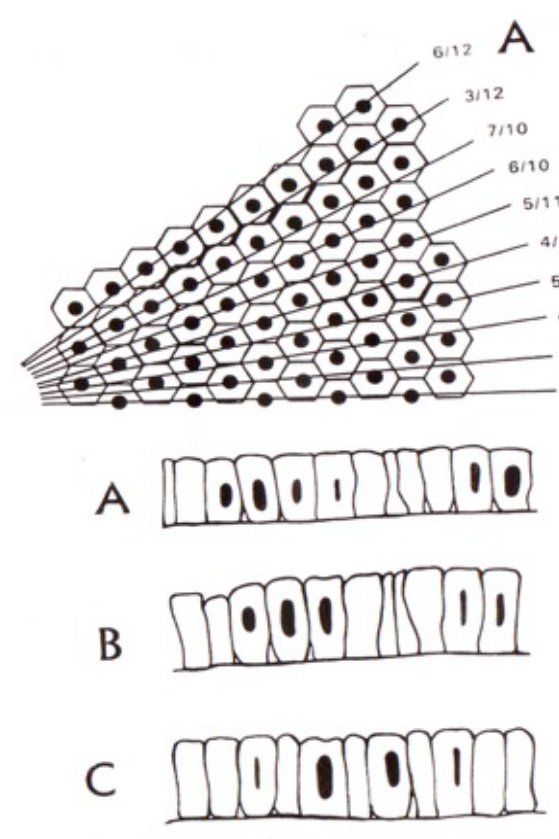

\section{Virchows Archiv 424: 301, 1994 422: 459,1993}

Figure 2. This is an 'idealised' epithelial array. The epithelial and nuclear dimensions have been carefully drawn to scale based on transmission and scanning EM data. The lines represent random sectioning planes through the epithelium. It is important to note that, on average, only $~ 50 \%$ of nuclei appear in any section. A, B, C are selected cuts, imaginatively reproduced below as they would appear in histological section. In those three representations, the high numbers of "lost" enterocyte nuclei can be appreciated, but which erroneously increases the so-called IEL count to almost double its correct (or absolute) number. We revealed that error elsewhere [2]

I have never seen any reference to the important paper of Guix and Whitehead, in which similar conclusions to my own data were offered [10]. Likewise, I know of no paper in which their simplified grid was used, which would have yielded accurate counts as well as consistent data, and which therefore could have satisfied Lerner \& Matthias' desire for one kind of systematic approach. Therefore, Pena's assertion [11] that Ferguson's erroneous method is 'a simple quantitation' needs considerable qualification. And while he quotes our papers [2,9], he fails to draw the obvious conclusions. Neither do I understand the purpose of his quoting Ferguson's data which showed villous infiltrations within $24 \mathrm{hr}$ in five challenged adult patients. We demonstrated with our technique considerable lymphocytic infiltration of villi within $12 \mathrm{hr}$ of ingesting a small challenge dose of $1 \mathrm{gm}$ Frazer's \#3 [12]. Finally, I was never able to understand why everyone was content to attempt capturing one moving target (IEL) by reference to another inconstant reference (the changing epithelium)! That is why I settled for referring all measurements to a constant test area of muscularis mucosae, thus to overcome that and other technical drawbacks.

To continue. Thin tissue sections contain not whole structures (ie lymphocytes) but sectioned profile-discs, so that "counting" is no longer a simple numerical task, as elaborated by Weibel [13]. These profile-discs occupy the thickness of the tissue section, but their actual 3dimensional size (presumptive mean diameter, d) must also be taken into account producing, with the nominal 
section thickness (t), what is termed the effective section thickness, EST: that is, $(\mathrm{t}+\mathrm{d} \mu \mathrm{m})$. But that is not a problem, since pre-calculated values can be employed in making accurate, reproducible counts. All that is required is that the spacing between sections must exceed EST and that numbers are accumulated along a measured length of muscularis mucosae [14]. Nothing could be simpler. The real skill is converting those profile-density counts back into real structures, as they existed in the tissue before sectioning took place: that is, to obtain absolute counts independent of other constraints such as enterocyte nuclei [9].

My perception is that not many people know about, have understood, and even overcome the underlying technical problems involved. The word "morphometry" needs to recognised as the basis of a new approach, and not dismissively used as a term of derision. With current machines for morphometric analysis, these more stringent methods could easily be employed in routine labs. The techniques are easily acquired, but they do need a little time to complete. I used to arrive in my lab at around 0530hrs, so to accomplish some effective measuring before the telephones began ringing at 9 o'clock: there are differences, however, between pure research and clinical accomplishment.

But some revisionary change is required, but whether it is necessary to employ flow cytometry or other highly sophisticated techniques, is questionable. It is easy for those accustomed to working in university-based hospitals and their ancillary research laboratories to immediately think of recruiting these refined tools and their skilled personnel. But that strategy is hardly relevant to those working in less refined circumstances, or in parts of the world where that kind of assistance remains only but a pipe-dream. It is necessary that this requirement is borne in mind. And, it should still be emphasised that simplified morphometric techniques are available and well-tried. They just need to be adopted by everyone in order to achieve a standardised methodological approach.

And that's what really counts.

\section{References}

[1] Siriweera E, Qi Z, Young J. Validity of intraepithelial lymphocyte count in diagnosis of celiac disease: a histopathological study. International Journal of Celiac Disease. 2015;3:156-158.

[2] Crowe P, Marsh MN. Morphometric analysis of small intestinal mucosa. VI: Principles in enumerating intra-epithelial lymphocytes. Virchows Archiv 1994;424:301-306.

[3] Marsh MN. Defining 'coeliac': Oslo accord - or not? Gut 2013;62:1669-1770.

[4] Lerner A, Matthias T. Intraepithelial lymphocyte normal cut-off level in celiac disease: the debate continues. International Journal of Celiac Disease 2016;4:4-6.

[5] Marsh, MN. Gluten, major histocompatibility complex, and the small intestine: a molecular and immunobiologic approach to the spectrum of gluten sensitivity ('celiac sprue'). Gastroenterology 1992;102:330-354.

[6] Leffler D, Vanga R, Mukherjee R. Mild enteropathy in celiac disease: a wolf in sheep's clothing. Gut 2013;11:259-261.

[7] Ludvigsson J, Bai J, Biagi F, Card T, Ciacci C, Ciclitira P, et al. Diagnosis and management of adult celiac disease: guidelines from the British Society of Gastroenterology. Gut 2014;63:12101228.

[8] Rostami K, Aldulaimi D, Holmes G, Johnson MW, Robert M, Srivastava A, et al. Microscopic enteritis: The Bucharest Consensus. World J Gastroenterol 2015;21:2593-2604.

[9] Crowe P, Marsh MN. Morphometric analysis of small intestinal mucosa: IV. Determining cell volumes. Virchows Archiv A Path Anat Histopathology 1993;422:459-466.

[10] Guix M, Skinner J, Whitehead R. Measuring intraepithelial lymphocytes, surface area, and volume of lamina propria in the jejuna mucosa of celiac patients. Gut 1979;20:275-278.

[11] Pena S. Counting intraepithelial lymphocytes. Immunochemistry and Flow Cytometer are necessary new steps in the diagnosis of celiac disease. International Journal of Celiac Disease 2016;4: 7-8.

[12] Marsh MN, Loft D, Garner V, Gordon D. Time/dose responses in celiac mucosae to graded oral challenges with Frazer's Fraction III (FF3) to gliadin. Eur J Gastroenterol Hepatol 1992;4:667-674.

[13] Weibel ER. Stereological Methods. [Vol. I]. New York [NY]: Academic Press 1979.

[14] Marsh MN, Crowe P, Moriarty K, Ensari A. Morphometric analysis of intestinal mucosa: The measurement of volume compartments and cell volumes in human intestinal mucosa. In: Marsh MN (ed), Celiac Disease: Methods \& Protocols (Methods in Molecular Medicine \#41). Totowa [NJ]: Humana Press 2000;125-145. 